\title{
Anisotropic Orientational Motion of Molecular Adsorbates at the Air-Water Interface
}

\author{
David Zimdars, ${ }^{\dagger}$ J. I. Dadap, ${ }^{\dagger}, \stackrel{+}{ }$ Kenneth B. Eisenthal, ${ }^{*}, \dagger$ and T. F. Heinz ${ }^{\S}$ \\ Department of Chemistry and Department of Physics, Columbia University, New York, New York 10027
}

Received: November 18, 1998

\begin{abstract}
The ultrafast orientational motions of coumarin 314 (C314) adsorbed at the air/water interface were investigated by time-resolved surface second harmonic generation (TRSHG). The theory and method of using TRSHG to detect both out-of-plane and in-plane orientational motions are discussed. The interfacial solute motions were found to be anisotropic, with differing out-of-plane and in-plane reorientation time constants. This report presents the first direct observation of in-plane orientational motion of a molecule (C314) at the air/water interface using TRSHG. The in-plane reorientation time constant is $600 \pm 40 \mathrm{ps}$. The out-of-plane reorientation time constant is $350 \pm 20$ ps. The out-of-plane orientational motion of C314 is similar to the previous results on rhodamine $6 \mathrm{G}$ at the air/water interface which indicated increased interfacial friction compared with bulk aqueous solution. The surface reorientation times are 2-3 times slower than the bulk isotropic orientational diffusion time.
\end{abstract}

\section{Introduction}

The physical and chemical properties of interfaces are of great importance to fundamental science, medicine, and technology. ${ }^{1-8}$ Intrinsic to the interface is the asymmetrical environment experienced by the chemical species at the interface,, 910 whether they are in the form of a solid or a molecular species, as in a gas or liquid, or are interface charges made up of electrons or ions. It is this interfacial asymmetry, which is absent in the bulk media, that determines the orientational structure, ${ }^{11}$ chemical composition, polarity, ${ }^{12,13}$ and transport properties of the interface. These factors, in turn, determine static properties such as interfacial chemical equilibria, ${ }^{14} \mathrm{pH},{ }^{15}$ and phases of longchain amphiphiles, ${ }^{11}$ as well as the dynamics of molecular motions, ${ }^{16,17}$ energy relaxation, ${ }^{18}$ and chemical change. Exploring interfacial orientational motion provides information important to such processes such as the rate of transport and the reactive stereochemistry of species at the interface.

Even the apparently simple example of the air/water interface shows complex properties in comparison to the interior of liquid water. Many types of molecules dissolved in water will gather in excess at the interface to lower the surface tension. Inside the water bulk, these dissolved molecules would rotate and diffuse in all directions. In comparison, the motions of a solute molecule at the air-water interface are restricted by an asymmetric surface potential energy. One end of the molecule may prefer to stick down into the water, while the other end may prefer to stick up into the air. This asymmetric surface environment can result in anisotropic orientational motion at the air/water interface. Rotational motions within the plane of the interface are no longer equivalent to rotational motions out of the plane of the interface.

In this investigation, time-resolved surface second harmonic generation (TRSHG) was used to probe the orientational motions

* To whom correspondence should be addressed. E-mail-eisenth@ chem.columbia.edu.

Department of Chemistry.

$\doteqdot$ Current address: Department of Physics, Columbia University, New York, NY 10027.

$\S$ Department of Physics. of coumarin $314(\mathrm{C} 314)$ at the air/water interface. A second harmonic ( $\mathrm{SH})$ signal is generated only by the chosen interfacial adsorbate (see refs 3 and 19 for reviews of SH spectroscopy). There is no interference from the bulk solute, since $\mathrm{SH}$ is electric dipole forbidden by symmetry in isotropic bulk media. $3,8,20,21$ Only those probe molecules present at the interface where inversion symmetry is broken can contribute to the $\mathrm{SH}$ signal.

The surface specificity of the SH signal gives TRSHG a great advantage over other spectroscopic methods sensitive to orientational motion. Conventional time-resolved fluorescence can typically be used only when the probe is so insoluble that it is present chiefly at the interface..$^{22-25}$ Occasionally, one of the bulk media has a favorable index of refraction to allow the fluorescence to be probed by total internal reflectance (TIR). ${ }^{24-27}$ Unfortunately, TIR is not rigorously surface specific since the evanescent wave probes hundreds of molecular layers $(\sim 1000$ $\AA$ ) beyond the true surface.

TRSHG has been used to study molecular dynamics of molecules at the air/quartz $\mathrm{z}^{26,28}$ and the liquid/quartz $\mathrm{z}^{29}$ interfaces. Rotational relaxation has been studied at the air/water interface $^{16,17}$ as well as processes such as energy transfer and photoisomerization ${ }^{18,30-32}$ among others.

Previously, we have used TRSHG to study the rotational motion of rhodamine $6 \mathrm{G}(\mathrm{Rh} 6 \mathrm{G})$ at the air/water interface. ${ }^{16}$ The results of our earlier study on Rh6G showed that the orientational out-of-plane motions are slower at the interface than in the bulk, whereas others have found that for eosin B the interfacial out-of-plane motions are faster in comparison to orientational motion in bulk water. ${ }^{17}$ In the previous work on Rh6 $6 \mathrm{G}^{16}$ and on eosin $\mathrm{B},{ }^{17}$ only the out-of-plane motions were detected. In the present study on coumarin 314, we detect both the in- and out-of-plane molecular motions and can explicitly discriminate the in-plane orientational motion.

This study represents the first direct observation of in-plane orientational motion of molecules at the water/air interface using TRSHG. With respect to the out-of-plane orientational motions, the results are similar to the previous study of Rh6G at the air/ water interface which showed that for these molecules there is greater friction at the air/water interface than in bulk water. 
A

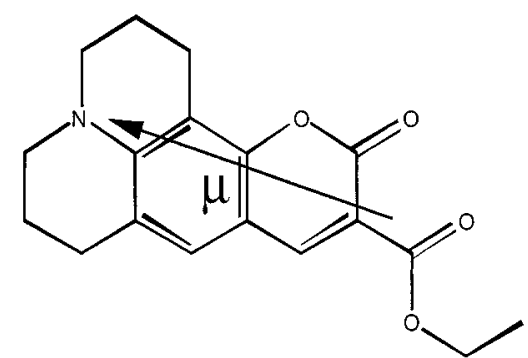

Coumarin 314

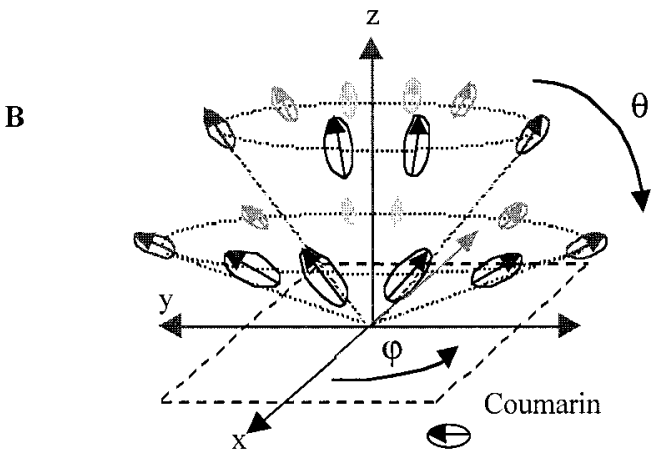

Figure 1. (A) Coumarin 314. The arrow shows the direction of the permanent dipole moment and the transition dipole moment of the $S_{0}$ to $S_{1}$ transition and is taken to be the reference axis of the molecule. (B) The distribution probability of $\mathrm{C} 314$ at the air/water interface. The angle $\Theta$ is the angle out-of-plane from normal, and $\varphi$ is the in-plane projection angle around the $z$-axis.

In addition to molecular rotation, we observed a component to the TRSHG decay attributable to electronic excited-state solvation. To our knowledge, little is known about the ultrafast solvation dynamics of molecules at liquid interfaces. In excitedstate solvation, the interfacial water solvent shell around the solute relaxes in reaction to the change in permanent dipole moment upon excitation of the solute. The excited-state contribution to the SH changes on the femtosecond time scale as the excited-state spectra shifts in wavelength. We have found that the solvent dynamics occur 2 orders of magnitude faster than the coumarin 314 solute orientational motion. Thus, the solvation component can be easily separated from rotational dynamics.

\section{Theoretical Considerations}

\subsection{Introduction to Time-Resolved Surface Second Har-} monic Generation. TRSHG is a form of pump-probe spectroscopy where surface solute molecules are first resonantly excited by an optical pump pulse and then monitored at a later time by detecting the second harmonic generated by a reflected optical probe. While the pump may excite solute beyond the surface, the technique only monitors the solute in the surface layer due to the surface specificity of SH. In addition to the solute population, orientational motions can be observed because the SH signal strongly depends on the orientation of the solute. As the solute reorients in time, the probe $\mathrm{SH}$ signal changes.

The probe frequency is usually chosen to be two-photon resonant with the origin electronic transition of the adsorbant $\mathrm{S}_{0}-\mathrm{S}_{1}$ (the states will be denoted "g" and "e", respectively). The reflected second harmonic field amplitude, $E_{2 \omega}$, is proportional to the surface nonlinear susceptibility, $\chi^{(2)}$, which in turn is proportional to the number of adsorbates per unit area, $N$, and the orientational average of the molecular hyperpolariz- abilities, $\left\langle\alpha^{(2)}\right\rangle$ (for brevity the direction cosine matrixes have been omitted). At equilibrium (before pumping or well after pumping), all molecules are in the ground state. The probability distribution of finding a ground-state adsorbate with out-of-plane angle $\theta$ and in-plane angle $\varphi$ is $\rho_{\mathrm{g}}(\theta, \varphi ; t<0)$. The nonlinear polarization $P_{2 \omega}$ before pumping is

$$
P_{2 \omega}(t<0)=E_{\omega}^{2}\left\langle\alpha_{\mathrm{g}}^{(2)} N_{\mathrm{g}}\right\rangle_{\rho_{\mathrm{g}}(\theta, \varphi ; t<0)}
$$

It is this ground-state orientational average from which the pump pulse photoselects an anisotropic subset of molecules in a manner that makes the recovery dynamics of the probed second harmonic sensitive to orientational motions. By choosing the pump polarization, the photoselection will yield dynamics dependent on both in- and out-of-plane motions or out-of-plane motions alone (see Figure 1B). The distribution of molecules excited by the pump pulse at $t=0$ will be denoted $\rho_{\mathrm{e}}(\theta, \varphi ; t=0)$. Initially, the number of excited molecules oriented at $\theta, \varphi$ will be proportional to $N_{\mathrm{e}} \rho_{\mathrm{e}}(\theta, \varphi ; t=0)$ with $N_{\mathrm{e}}$ being the total number of interface excited molecules at $t=0$. The number of groundstate molecules oriented at $\theta, \varphi$ is proportional to $N \rho_{\mathrm{g}}(\theta, \varphi ; \mathrm{t}<0)$ $-N_{\mathrm{e}} \rho_{\mathrm{e}}(\theta, \varphi ; t=0)$, where $N$ is the total number of molecular adsorbates. After the initial excitation, the time-dependent distributions $\rho_{\mathrm{e}}(\theta, \varphi ; t)$ and $\rho_{\mathrm{g}}(\theta, \varphi ; t)$ will evolve as the excitedand ground-state molecules orientationally diffuse to their equilibrium orientational distributions.

If at time $t=0$ the adsorbant is pumped by a one-photon resonant polarized pump pulse (Figure 2), the probe $\mathrm{SH}$ polarization at time $t$ is given by

$$
\begin{aligned}
& P_{2 \omega}(t \geq 0)=E_{\omega}{ }^{2}\left\langle\alpha_{\mathrm{g}}^{(2)}\left(N-N_{\mathrm{e}}(t)\right)\right\rangle_{\rho_{\mathrm{g}}(\theta, \varphi ; t)}+ \\
& E_{\omega}{ }^{2}\left\langle\alpha_{\mathrm{e}}^{(2)} N_{\mathrm{e}}(t)\right\rangle_{\rho_{\mathrm{e}}(\theta, \varphi ; t)}
\end{aligned}
$$

The orientational averages \langle\rangle are time dependent because of the evolution of the orientational probability function $\rho(\theta, \varphi ; t)$. The populations of ground- and excited-state adsorbates change with the excited-state lifetime as well.

Population recovery, orientational motion, and electronic excited-state solvation are well separated in time, allowing a single pump-probe decay to have resolvable kinetics on the nanosecond, picosecond, and femtosecond time scales corresponding to the excited-state lifetime, reorientational time, and solvation time, respectively. In addition to orientational relaxation, which we will see occur on the time scale of hundreds of picoseconds, we have observed excited-state solvation dynamics $(<1 \mathrm{ps})$ and excited-state population recovery $(\sim 5 \mathrm{~ns})$. Because the solvation time scale is so much shorter and the population recovery time scale so much longer, the orientational dynamics can be easily separated by only considering the pump-probe data from $\sim 2$ ps to $\sim 1 \mathrm{~ns}$.

2.2. Dependence of Second Harmonic on Orientational Motions. In the following sections, the sensitivity of SH to orientational motions will be shown more explicitly. The second harmonic signal field generated at the interface is proportional to the square root of the experimentally measured intensity, $I(2 \omega)=(E(2 \omega))^{1 / 2}$. The signal SH field is proportional to the induced second-order nonlinear polarization, $E(2 \omega) \propto P^{(2)}(2 \omega)$. The SH polarizations with components $i, j$, are more succinctly written as the probe electric field acting twice times the secondorder nonlinear polarizability,

$$
P_{i}^{(2)}(2 \omega ; t)=E_{j}(\omega) E_{k}(\omega) \chi_{i, j, k}^{(2)}(2 \omega, \omega, \omega ; t)
$$

where the subscripts $i, j, k$ are determined by the polarization 


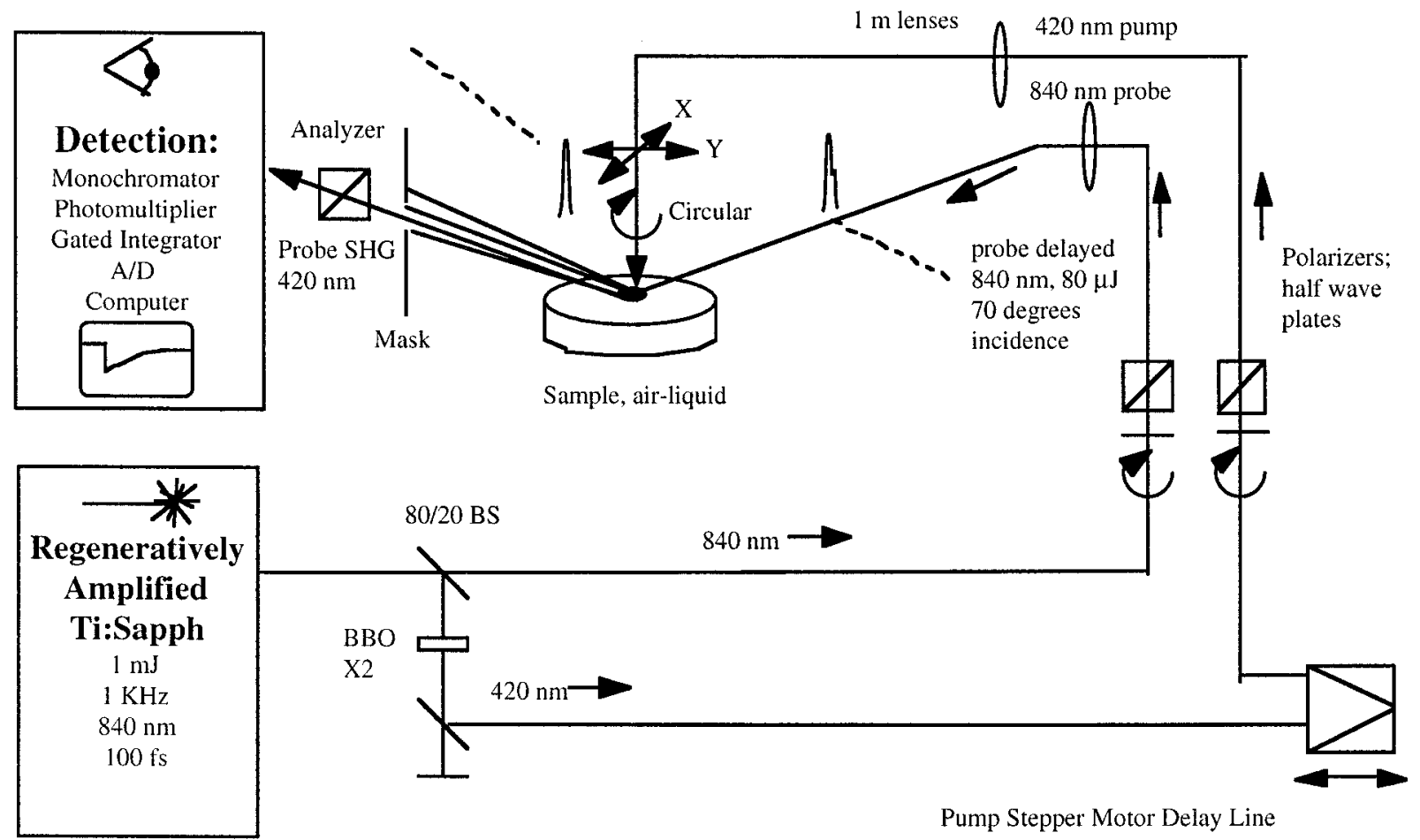

Figure 2. Time-resolved second harmonic generation pump probe setup for detection of orientational motion. The experimental setup is described in detail in the text.

of the analyzed SH and probe fundamental (subsequently for brevity, the frequency indices $2 \omega, \omega, \omega$ will be omitted). All of the dynamics of eq 2 will be included in this tensor. The surface second-order nonlinear susceptibility tensor for a groundstate ensemble of $\mathrm{C} 314$ at the air/water interface can be expressed as ${ }^{1,33,34}$

$$
\chi_{i j k_{\mathrm{g}}}^{(2)}(\mathrm{eq})=N_{\mathrm{g}} \sum\left\langle T_{l m n}^{i j k}\right\rangle_{\mathrm{g}} \alpha_{l m n_{\mathrm{g}}}^{(2)}
$$

where $T_{l m n}^{i j k}$ is the direction cosine matrix that transforms the laboratory frame $(i, j, k)$ into the molecular frame $(l, m, n), N_{\mathrm{g}}$ is the number of ground-state molecules at the surface at equilibrium, $\alpha_{l m n_{\mathrm{g}}}^{(2)}$ is the molecular hyperpolarizability for the $l$, $m, n$ axes, and \langle\rangle denotes the orientational ensemble average. By symmetry, only the elements $\chi_{z z z}^{(2)}, \chi_{z x x}^{(2)}$, and $\chi_{x z x}^{(2)}$ are nonzero at an interface (the $X$ and $Y$ axes are equivalent for an isotropic surface). Experimentaly, the elements $\chi_{z x x}^{(2)}$ and $\chi_{x z x}^{(2)}$ can be isolated by the proper choice of fundamental and $\mathrm{SH}$ analysis polarizations.

C314 is shown in Figure 1A, where the molecular $\zeta$-axis is taken to be along the transition dipole moment $\mu_{\text {ge }}$ of the electronic origin at $420 \mathrm{~nm}$. This $\zeta$-axis will be considered the orientational reference axis. The number of nonzero hyperpolarizability elements $\alpha_{l m n}^{(2)}$ contributing to eq 4 is determined by the symmetry of the molecule.

For the purposes of analysis of the time-resolved SH signal, our measurements indicate that the symmetry of C314 can be approximated as uniaxial. Uniaxial symmetry predicts that $\chi_{x z x}^{(2)}$ $=\chi_{z x x}^{(2)}$. Experimental data was taken chiefly for $\chi_{x z x}^{(2)}$; however, the equivalence of the $\chi_{z x x}^{(2)}$ and $\chi_{x z x}^{(2)}$ time-resolved signal was verified experimentally to show that this approximation is valid.

There is only one hyperpolarizability element in the ground state and excited state for uniaxial symmetry. Since the only strong SH resonance enhancement for C314 is the two-photon resonance of the ground and lowest excited state, we can make the approximation that C314 is nearly a two-level system. In this case, the ground- and excited-state uniaxial hyperpolarizabilities, $\alpha_{\mathrm{g}}^{(2)} \zeta \zeta \zeta$ and $\alpha_{\mathrm{e}}^{(2)} \zeta \zeta \zeta$, are parallel to the transition dipole moment $\mu_{\mathrm{ge}}$ along the reference axis $\zeta$. For a two-level system, the microscopic hyperpolarizabilities are opposite in sign, $\alpha_{\mathrm{e}}^{(2)} \zeta \zeta \zeta_{\zeta}=-\alpha_{\mathrm{g}}^{(2)} \zeta \zeta \zeta .^{20}$ However, because the excited-state solvation can shift the ground/excited-state energy separation, the two-photon resonance of the excited state with the probe pulse, the relationship for $\mathrm{C} 314$ is only approximate, $\alpha_{\mathrm{e}}^{(2)} \xi \xi \xi \approx$ $-\alpha_{\mathrm{g}}^{(2)} \zeta \zeta \zeta$. Note that, for a uniaxial molecule, it is assumed that the average over the angle $\Psi$ about the reference axis (not shown) can be replaced by some constant average value.

The orientational probability distribution and angular notation is shown in Figure 1B. The out-of-plane angle $\theta$ subtends the molecular $\zeta$ reference axis and the $z$-axis normal to the surface plane. The in-plane angle $\varphi$ subtends the projection of the reference axis $\zeta$ in the surface $x-y$ plane and the $x$-axis. The in-plane $\varphi$ angular distribution is isotropic at equilibrium because the in-plane intermolecular forces are isotropic. However, the asymmetry in forces normal to the interface, e.g., air above and water below the interface, is responsible for the high degree of orientation in the adsorbates out-of-plane angle $\theta$. This orientational distribution is depicted schematically in Figure $1 \mathrm{~B}$ as the $\mathrm{C} 314$ molecules lying between two cones, i.e., principally oriented between the $\theta$ values of the two cones. For $\mathrm{C} 314$ at the air/water interface, the out-of-plane distribution in the ground state was measured to be peaked near $80^{\circ}$ by the null angle technique. ${ }^{1}$

Because C314 has a large change in dipole moment on excitation, the equilibrium $\theta_{\max }$ is not necessarily the same for the ground and excited states. The differing electrostatic interactions with the solvent molecules in the interface region could drive the excited state to a different out-of-plane angle $\theta$, and $\rho_{\mathrm{e}}(\theta, \varphi, t \gg 0) \neq \rho_{\mathrm{g}}(\theta, \varphi, t<0)$ where $\mathrm{t} \gg 0$ means a sufficiently long time such that the excited states have reached their equilibrium orientational distribution. A difference in the 


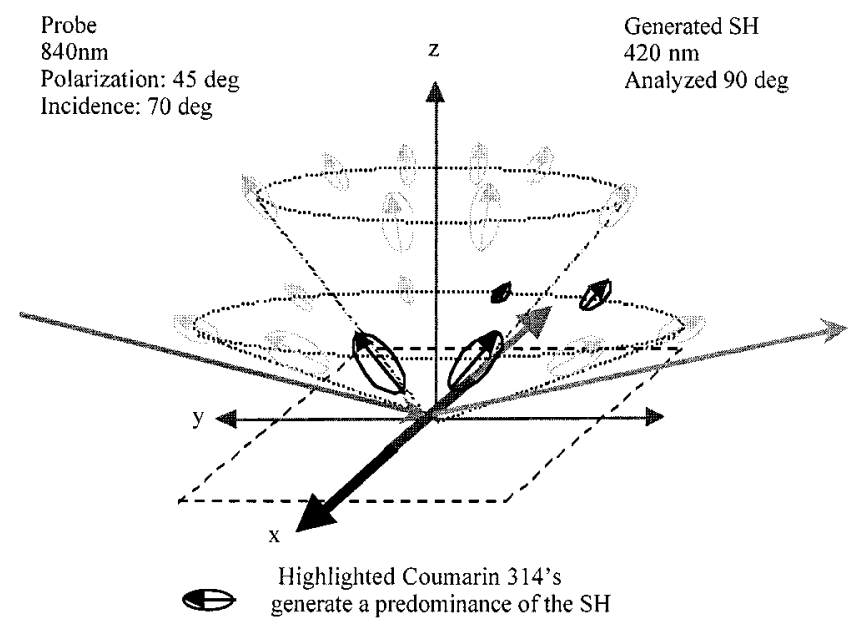

Figure 3. Schematic of SH from $\mathrm{C} 314$ at the air/water interface and detection of the $\chi_{x z x}^{(2)}$ tensor element. Darkened C314's contribute most strongly to the orientational average.

out-of-plane angle between the ground and excited state was observed previously with Rh6G at the air/water interface. ${ }^{16,35}$

The direction cosine matrix element transforming the single uniaxial hyperpolarizability $\alpha_{\mathrm{g}}^{(2)} \xi \xi \xi$ into the lab frame is $\sin ^{2} \theta \cos \theta \cos ^{2} \varphi$ for both $\chi_{x z x}^{(2)}$ and $\chi_{z x x}^{(2)}$. For brevity, only $\chi_{x z x}^{(2)}$ will be denoted in the following equations and discussion. However, in all cases $\chi_{z x x}^{(2)}=\chi_{x z x}^{(2)}$. The susceptibility $\chi_{x z x_{\mathrm{g}}}^{(2)}$ for the ground state can be written as,

$$
\chi_{x z x_{\mathrm{g}}}^{(2)}(\mathrm{eq})=N_{\mathrm{g}} \alpha_{\mathrm{g} \zeta \zeta \zeta}^{(2)}\left\langle\sin ^{2} \theta \cos \theta \cos ^{2} \varphi\right\rangle_{\rho_{\mathrm{g}}(\theta, \varphi ; t<0)}
$$

and explicitly as

$$
\begin{array}{r}
\chi_{x z x_{\mathrm{g}}}^{(2)}(\mathrm{eq})=N_{\mathrm{g}} \alpha_{\mathrm{g}}^{(2)} \xi \xi \zeta \int_{0}^{2 \pi \pi} \int_{0} \sin ^{2} \theta \cos \theta \cos ^{2} \varphi \\
\rho_{\mathrm{g}}(\theta, \varphi ; t<0) \sin \theta \mathrm{d} \theta \mathrm{d} \varphi
\end{array}
$$

The orientational average in eq 6 weights the contribution of the microscopic hyperpolarizability $\alpha_{\xi \zeta \xi}^{(2)}$ to the macroscopic susceptibility $\chi_{x z x}^{(2)}$ by the orientational probability distribution in angles $\theta$ and $\varphi$ for a surface ensemble of C314 and the direction cosine matrix element $\sin ^{2} \theta \cos \cos ^{2} \varphi$. Figure 3 illustrates how the result of this average weights different angles $\theta$ and $\varphi$. Those C314 molecules with molecular axes $\xi$ more nearly in-plane (larger angle $\theta$ ) are weighted greater in $\chi_{x z x}^{(2)}$ due to the $\sin ^{2} \theta \cos \theta$ portion of the direction cosine matrix element. Likewise, those $\mathrm{C} 314$ molecules that lie more nearly along the lab frame " $X$ "-axis (small angle $\varphi$ ) are also weighted greater in $\chi_{x z x}^{(2)}$ due to the $\cos ^{2} \varphi$ portion of the direction cosine matrix element. However, those molecules with their $\xi$ axis oriented closer to the laboratory " $Y$ "-axis, $\varphi \approx \pi / 2$, or with $\theta$ near $0^{\circ}$ contribute less to $\chi_{x z x}^{(2)}$.

2.3. Time Dependence Upon Pumping. The strategy to detect orientational motion is to selectively excite C314 molecules with a polarized pump pulse, thereby changing the orientational distribution of molecules and thus changing the orientational average of the $\chi_{x z x}^{(2)}$ element. The ensuing timedependent changes in the orientations of the remaining groundstate molecules and the photogenerated excited-state molecules will be detected by the probe as the molecules move into or out of the predominately weighted angles of $\theta$ and $\varphi$ as they move to their equilibrium orientations. The time-dependent $\chi_{x z x}^{(2)}(t)$ element can be written as the sum of the ground- and excited-state parts,

$$
\chi_{x z x}^{(2)}(t)=\chi_{x z x_{\mathrm{g}}}^{(2)}(t)+\chi_{x z x_{\mathrm{e}}}^{(2)}(t)
$$

Those ground-state molecules that were not photoexcited reorient in the process of returning to the equilibrium groundstate distribution $\rho_{\mathrm{g}}(\theta, \varphi, t<0)$, whereas the pumped excited-state molecules orientationally diffuse toward whatever may be the equilibrium excited-state distribution $\rho_{\mathrm{e}}(\theta, \varphi, t \gg 0)$. Since the excited-state lifetime of $\mathrm{C} 314$ is an order of magnitude longer than the measured orientational dynamics, it is not necessary to consider the contribution of the ground states that were initially photoexcited. The contribution of population relaxation (and excited-state solvation) to the dynamics will be dropped in further equations due to the separation in time scales.

The TRSHG data will be analyzed in terms of in- and outof-plane motion without a complete evaluation of the $\chi^{(2)}$ elements appearing in eq 7. The time-dependent probability distributions that govern the time evolution of ground- and excited-state molecules, which are contained in $\chi_{x z x}^{(2)}(t)$, depend on the pumping geometry, the polarization of the pump light, the ground- and excited-state equilibrium distributions, and the ensuing orientational motions at the interface. While we know a-priori the pumping geometry and polarization, an explicit model of the ground- and excited-state orientational equilibrium distributions and orientational motion must be assumed or inferred from other data. We are currently developing a model which contains the ground- and excited-state contributions to eq 7. The equilibrium orientation of the ground-state molecules, i.e., the distribution before the pump light, can be measured assuming a narrow distribution of ground-state out-of-plane orientations. The in-plane orientations of the ground- and excited-state molecules are isotropic in the interfacial $(X-Y)$ plane because there are no anisotropic orienting forces in the plane.

For the heuristic purpose of illustrating how the pumping geometry and polarization can discriminate the two types of motions, the contribution of the excited state to eq 7 will be presented in greater detail. The ground-state contribution is analogous, but for the sake of brevity it will be omitted since the evaluation of a more explicit equation is not necessary for this work.

2.4. Excited-State Orientational Motions. At $t=0$, the probability of photoexciting a molecule whose transition dipole moment $\vec{\mu}$ is oriented at angles $\theta, \varphi$ with respect to the laboratory axes will be denoted $\sigma \vec{\mu} \cdot \vec{E}^{2}(\theta, \varphi)$ where $\vec{E}$ is the electric field of the pump and $\sigma$ is a collection of all constants associated with photoexcitation. This excitation probability is projected onto the ground-state distribution $\rho_{\mathrm{g}}(\theta, \varphi, t<0)$ so that at $t=0 \rho_{\mathrm{e}}(\theta, \varphi ; t=0)=\sigma|\vec{\mu} \cdot \vec{E}|^{2}(\theta, \phi) \rho_{\mathrm{g}}(\theta, \varphi ; t<0)$ and

$$
\begin{aligned}
& \chi_{x z x_{\mathrm{e}}}^{(2)}(0)=N_{\mathrm{e}} \alpha_{\mathrm{e}}^{(2)} \zeta \zeta \zeta \int_{0}^{2 \pi \pi} \int_{0}^{2 \pi} \sin ^{2} \theta \cos \theta \cos ^{2} \\
& \varphi|\vec{\mu} \cdot \vec{E}|^{2}(\theta, \varphi) \rho_{\mathrm{g}}(\theta, \varphi ; t<0) \sin \theta \mathrm{d} \theta \mathrm{d} \varphi
\end{aligned}
$$

For times $t \gg 0$, where the excited molecules have redistributed to the equilibrium excited-state distribution $\rho_{\mathrm{e}}(\theta, \varphi, t \gg 0)$, then

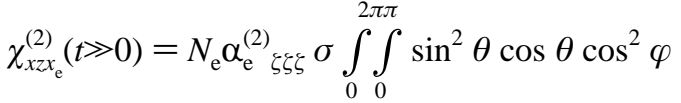

$$
\begin{aligned}
& \rho_{\mathrm{e}}(\theta, \varphi ; t \gg 0) \sin \theta \mathrm{d} \theta \mathrm{d} \varphi
\end{aligned}
$$


An evolution function $G_{\mathrm{e}}(\theta, \varphi ; 0, t)$ can be used to describe the reorientation from $\rho_{\mathrm{e}}(\theta, \varphi, t=0)$ to $\rho_{\mathrm{e}}(\theta, \varphi, t \gg 0)$ so that

$$
\begin{aligned}
& \chi_{x z x_{\mathrm{e}}}^{(2)}(t)=N_{\mathrm{e}} \alpha_{\mathrm{e}}^{(2)} \zeta \zeta \zeta \\
& \varphi \int_{0}^{2 \pi \pi} \int_{0}^{2 \pi} \sin ^{2} \theta \cos \theta \cos ^{2} \\
& \varphi|\vec{\mu} \cdot \vec{E}|^{2}(\theta, \varphi) \rho_{\mathrm{g}}(\theta, \varphi ; t<0) G_{\mathrm{e}}(\theta, \varphi ; 0, t) \sin \theta \mathrm{d} \theta \mathrm{d} \varphi
\end{aligned}
$$

Again, the evaluation of eq 10 requires the explicit form of $G_{\mathrm{e}}(\theta, \varphi ; 0, t)$ and $\rho_{\mathrm{e}}(\theta, \varphi ; t \gg 0)$ contained in $G_{\mathrm{e}}(\theta, \varphi ; 0, t)$ for $t \gg$ 0 . These functions depend on the model of orientational diffusion at the interface. However, eq 10 is useful in illustrating how the pumping polarization can select in- and out-of-plane orientational dynamics. The analogous form for $\chi_{x z x_{\mathrm{g}}}^{(2)}(t)$ contains a different evolution function $G_{\mathrm{g}}(\theta, \varphi ; 0, t)$ that contains the ground-state reorientation dynamics.

2.5. Pumping Polarization Selection of Orientational Dynamics. The pumping geometry was chosen such that the angle of incidence was normal to the air/water interface, $\theta=$ 0 . That is, the pump electric field was parallel to the $X-Y$ plane of the interface. Only the polarization of the electric field within the $X-Y$ plane was varied. Three different pumping polarizationst were used: circularly polarized in the $X-Y$ plane ("C"), linearly polarized along the $X$-axis (" $X$ "), and linearly polarized along the $Y$-axis ("Y"). In all cases, the pump intensity was kept an order of magnitude below the saturation level for C314 to avoid any distortion in the selected projection of the polarization. See Figure 4 for an illustration of the coordinate system.

The probability expressions for the excitation of a groundstate molecule oriented with angles $\theta$ and $\varphi$ with respect to the laboratory axes are

" $\mathrm{C}$ ” circularly polarized pumping:

$$
\left|\vec{\mu} \cdot \vec{E}_{\mathrm{C}}\right|^{2}(\theta, \varphi)=\mu^{2} E_{\mathrm{c}}^{2} \sin ^{2} \theta
$$

" $\mathrm{X}$ " pumping polarized along $x$-axis:

$$
\left|\vec{\mu} \cdot \vec{E}_{\mathrm{X}}\right|^{2}(\theta, \varphi)=\mu^{2} E_{\mathrm{X}}^{2} \sin ^{2} \theta \cos ^{2} \varphi
$$

"Y" pumping polarized along $y$-axis:

$$
\left|\vec{\mu} \cdot \vec{E}_{\mathrm{Y}}\right|^{2}(\theta, \varphi)=\mu^{2} E_{\mathrm{Y}}{ }^{2} \sin ^{2} \theta \sin ^{2} \varphi
$$

Where $E$ is the electric field amplitude of the pump light for the appropriate polarization.

2.5.1. Out-of-Plane Orientational Dynamics. The out-of-plane orientational dynamics may be isolated by pumping normal to the liquid plane with circularly polarized light "C", eq 11a. The reason for this is that the ground-state molecules are isotropically distributed in the interface plane and the amplitude of the circularly polarized pump light is also isotropic in the interface plane. This in-plane isotropy of molecules and pump light produces an in-plane isotropic distribution of excited molecules and remaining ground-state molecules. Because the probability of exciting a C314 with out-of-plane angle $\theta$ goes as $\sin ^{2} \theta$, the probability of exciting a $\mathrm{C} 314$ with a larger angle $\theta$ is greater than that of exciting a $\mathrm{C} 314$ with a smaller $\theta$. Because the circularly polarized pump induces an anisotropy only for the out-of-plane angle, $\theta$, the kinetics observed are due only to outof-plane motions.

The distribution excited by circularly polarized light is depicted in Figure 4A by highlighting those C314 on the lower "cone" with a large angle $\theta$. As discussed in section 2.2, those molecules with larger angle $\theta$ contribute more strongly to $\chi_{x z x}^{(2)}$ than those with small angle $\theta$. The measured SH signal will decrease after the pump pulse at $t=0$ due to the opposing
A

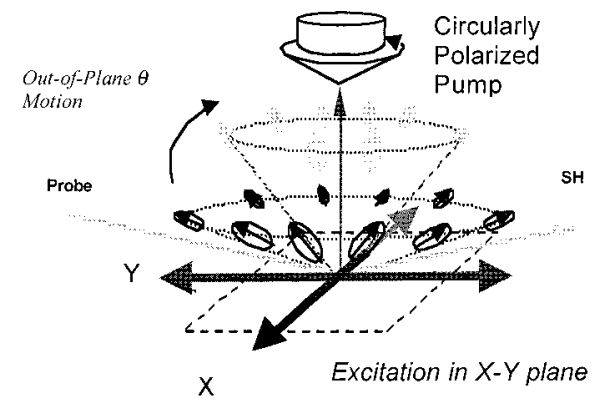

B

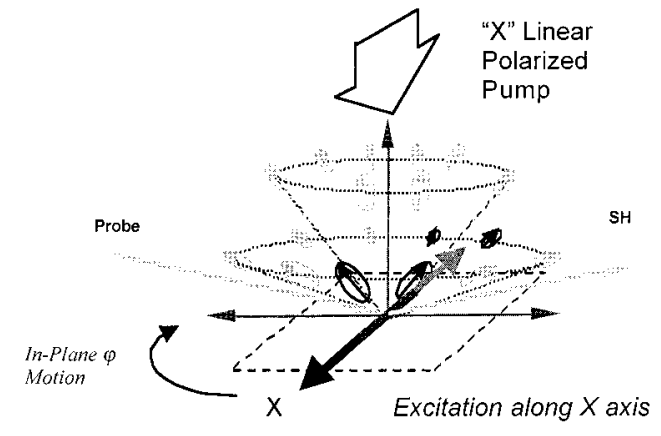

C

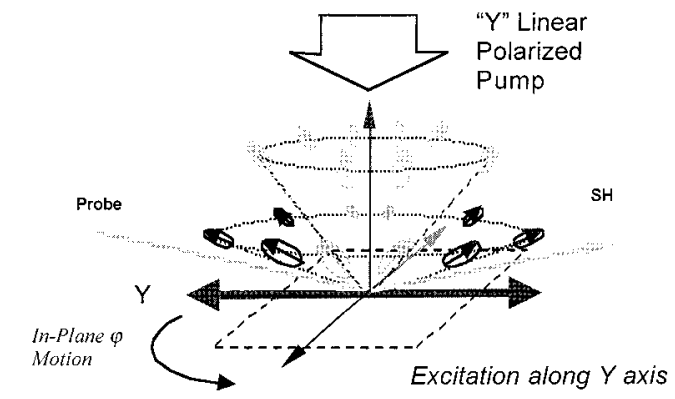

Figure 4. (A) Schematic showing the effect of normal incidence circularly polarized pumping. (B) Schematic showing the effect of pumping polarized along the $X$-axis. (C) Schematic showing the effect of pumping polarized along the $Y$-axis. The text describes how these pumping schemes discriminate in-plane and out-of-plane orientational motion.

contributions of the newly excited molecules and the remaining ground states. Because the excitation is not isotropic in the outof-plane angle $\theta$, the SH signal will recover with the out-ofplane reorientation time as the equilibrium distributions are regained in the ground and excited states. However, complete recovery of the SH signal to its baseline, i.e., its value at $t<0$, will not occur until the excited molecules relax to the ground electronic state.

2.5.2. In-Plane Orientational Dynamics. The scheme to differentiate in-plane from out-of-plane orientational motions relies on using linearly polarized pumping and the different contributions to $\chi_{x z x}^{(2)}$ of molecules oriented along the $X$-axis vs molecules oriented along the $Y$-axis. Unlike the circularly polarized pumping experiments, the ground- and excited-state populations are no longer isotropic in the $X-Y$ plane when the pump light is linearly polarized along the $X$ or $Y$ axes. As the populations of ground- and excited-state molecules along the $X$ and $Y$ axes reorient, their effects on $\chi_{x z x}^{(2)}$ differ and the $\mathrm{SH}$ probe detects in-plane kinetics.

The orientational distribution of $\mathrm{C} 314$ excited by pumping along the $X$-axis is depicted in Figure $4 \mathrm{~B}$ and is given by eq 11b. The in-plane excitation distribution for the $X$-axis is proportional to $\cos ^{2} \varphi$. As noted earlier in section 2.2, molecules oriented along the $X$-axis make a larger contribution to $\chi_{x z x}^{(2)}$ than those having their transition moments oriented along the 
$Y$-axis. Pumping along the $X$-axis will remove more groundstate molecules and create more excited-state molecules along the $X$-axis than for a circularly polarized pump pulse of equal intensity. Thus, the change in SH signal will be greater for the detection of the $\chi_{x z x}^{(2)}$ element, i.e., incident probe light polarized along $X$ and $Z$ and $\mathrm{SH}$ polarized along the $X$-axis. Note that the probability of exciting a C314 with out-of-plane angle $\theta$ is still $\sin ^{2} \theta$, and $X$-axis linear pumping will still detect outof-plane motions. The effect of $X$-axis linear pumping is to create an in-plane component to the TRSHG decay.

After X-polarized linear pumping, the orientational diffusion back to equilibrium will serve to reorient the excited states away from the $X$-axis to achieve isotropic orientation in the $X-Y$ plane. The remaining ground states likewise reorient back toward the $X$-axis, because of the deficiency of ground-state molecules due to the X-polarized pump light. As reorientation progresses, the $X$-axis excited-state molecules rotate away from the $\mathrm{X}$ orientation, and the ground-state molecules rotate toward the $X$ direction. Both the excited molecules orienting away from $X$ and the ground-state molecules orienting toward $X$ serve to increase $\chi_{x z x}^{(2)}$ and thus the SH signal. Ultimately, the linearly pumped orientational ensemble will reach the equilibrium orientational distribution, i.e., the in-plane distribution is isotropic in angle $\varphi$. This equilibrium distribution is identical to that reached by pumping with circularly polarized light. Thus, the linearly pumped SH will recover to the same level as reached by circularly polarized pumping.

The effect of $Y$-axis linear pumping is analogous to that of $X$-axis linear pumping but opposite in effect for the in-plane component. The orientational distribution of C314 excited by pumping along the $Y$-axis is illustrated in Figure 4C and given by eq 11c. For $Y$-axis pumping, the in-plane excitation distribution varies as $\sin ^{2} \varphi$. Given that the contribution to the $\chi_{x z x}^{(2)}$ tensor element by molecules along the $Y$-axis is minimal in comparison to the $X$-axis as described in section 2.2, the change in SH signal at $t=0$ will now be less in comparison to circularly polarized pumping since circularly polarized pumping pumps along the $X$ axis as well as the $Y$-axis.

The time evolution for $Y$ pumping can be described in a similar way as was done for $\mathrm{X}$ pumping. The orientational diffusion back to equilibrium will serve to reorient the excitedstate molecules away from the $Y$-axis to achieve isotropic orientation in the $X-Y$ plane. The remaining ground-state molecules likewise reorient back toward the $Y$-axis where there is a deficiency of ground-state molecules. Adding excited states into the $X$-axis and ground states into the $Y$-axis will serve to decrease the SH signal.

Since $\chi_{z x x}^{(2)}=\chi_{x z x}^{(2)}$ for a molecule with uniaxial symmetry (section 2.2), the change in $\chi_{z x x}^{(2)}$ with time due to orientational motions should be identical to that of $\chi_{x z x}^{(2)}$. The descriptions of the dependence of $\chi_{z x x}^{(2)}$ to circularly polarized pumping (section 2.5.1) and to linearly polarized pumping (section 2.5.2) are identical to those for $\chi_{x z x}^{(2)}$. It was verified experimentally that the kinetics of C314 at the air/water interface were the same for the $\chi_{z x x}^{(2)}$ and $\chi_{x z x}^{(2)}$ elements.

2.6. Isolation of In-Plane Orientational Dynamics. The in-plane orientational dynamics can be isolated from the outof-plane orientational dynamics without a specific model of orientational diffusion at the interface if the time-dependent excited- and ground-state distributions $\rho(\theta, \varphi ; t)$ are separable in angles $\theta$ and $\varphi$. That is, if $\rho(\theta, \varphi ; t)=\rho_{\theta}(\theta ; t) \rho_{\varphi}(\varphi ; t)$. In terms of eq 10 , the equilibrium distribution $\rho(\theta, \varphi ; t<0)$ and the time evolution function $G(\theta, \varphi ; 0, t)$ must be separable in addition to the pumping projection functions $|\vec{\mu} \cdot \vec{E}|^{2}(\theta, \varphi)$.

The pumping probability functions are separable as shown in eqs $11 \mathrm{a}-\mathrm{c}$. The equilibrium distribution functions $\rho_{\mathrm{g}}(\theta, \varphi ; t<0)$ and $\rho_{\mathrm{e}}(\theta, \varphi ; t \gg 0)$ are also separable since they must be isotropic in the in-plane angle $\varphi$, that is $\rho_{\varphi}(\varphi ; t \gg 0)=$ const. We make as a first approximation that the orientational motion described by $G(\theta, \varphi ; 0, t)$ can be separated in $\theta$ and $\varphi$. This assumption can be made for molecules adsorbed to a liquid interface that are oriented between a range of out-of-plane angles $\theta$ that is not wide. If the out-of-plane distribution is not too wide, the rate of in-plane orientational diffusion is not likely to vary greatly within the restricted values of $\theta$.

The time dependence of the susceptibility $\chi_{x z x}^{(2)}(t)$ in eq 7 can be expressed as the sum of the equilibrium value of the susceptibility plus a time-induced change,

$$
\chi_{x z x}^{(2)}(t)=\chi_{x z x}^{(2)}(\mathrm{eq})+\Delta \chi_{x z x}^{(2)}(t)
$$

where $\Delta \chi_{x z x}^{(2)}(t)$ gives the change of the susceptibility at time $t$ with respect to the equilibrium value $\chi_{x z x}^{(2)}(\mathrm{eq})$, that is, $\chi_{x z x}^{(2)}(\mathrm{eq})$ $=\chi_{x z x}^{(2)}(t<0)$.

The orientational dynamics in the ground and excited state are taken to be nearly equivalent. This equivalence of the ground- and excited-state dynamics is based on the observation that the out-of-plane kinetics observed in the circularly polarized pumping experiments yielded a single exponential decay. If the rotation times of the ground- and excited-state molecules were significantly different, the observed decay would have been nonexponential. The consequence of this approximate equality of ground- and excited-state orientational dynamics is that the averages in eq 12 can be written as \langle\rangle$_{\rho \theta(\theta ; t) \rho \varphi(\varphi ; t)}=\mathrm{c}_{\theta}(t) c_{\varphi}(\mathrm{t})$, the product of an out-of-plane $\theta$ function and an in-plane $\varphi$ function. The change in susceptibility is then

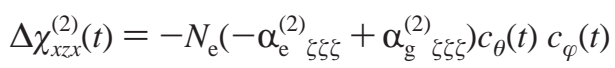

Note that the functions $c_{\theta}(t) c_{\varphi}(\mathrm{t})$ contain all of the orientational dynamics. For circular pumping $c_{\varphi: \text { circ }}(t)=$ const, whereas for " $\mathrm{X}$ " and " $\mathrm{Y}$ " linear pumping $c_{\varphi: \mathrm{X}}(t) \neq c_{\varphi: \mathrm{Y}}(t)$. These latter quantities $c_{\varphi: \mathrm{X}}(t)$ and $c_{\varphi: \mathrm{Y}}(t)$ are not equal because they make different contributions to the measured susceptibility element $\chi_{x z x}^{(2)}(t)$. However, in all cases $c_{\theta}(t)=c_{\theta: \text { circ }}(t)=c_{\theta: \mathrm{X}}(t)=c_{\theta: \mathrm{Y}}(t)$ because the projection of the molecular orientation $\theta$ onto the incident electric field is identically $\sin ^{2} \theta$ for all of these pumping polarizations.

The in-plane orientational dynamics may be isolated from the out-of-plane orientational dynamics by noting that the outof-plane component $c_{\theta}$ is identical for both $\mathrm{X}$ and $\mathrm{Y}$ pumping (see eqs 6 and 7). This component may be factored out by using the ratio

$$
\begin{array}{r}
\frac{\Delta \chi_{x z x: \mathrm{X}}(t)-\Delta \chi_{x z x: \mathrm{Y}}(t)}{\Delta \chi_{x z x: \mathrm{X}}(t)+\Delta \chi_{x z x: \mathrm{Y}}(t)}=\frac{\left[c_{\varphi: \mathrm{X}}(t)-c_{\varphi: \mathrm{Y}}(t)\right] c_{\theta}(t)}{\left[c_{\varphi: \mathrm{X}}(t)+c_{\varphi: \mathrm{Y}}(t)\right] c_{\theta}(t)}= \\
\frac{c_{\varphi: \mathrm{X}}(t)-c_{\varphi: \mathrm{Y}}(t)}{c_{\varphi: \mathrm{X}}(t)+c_{\varphi: \mathrm{Y}}(t)}
\end{array}
$$

This yields an expression which only contains in-plane orientational components and which can be used as a phenomenological representation of the in-plane orientational dynamics.

\section{Experimental Section}

The probe molecule chosen for this study was coumarin 314 (C314) (Figure 1A). C314 was obtained commercially (Acros) 
and used without further purification. All solution concentrations were measured by UV/vis spectroscopy. The solution $\mathrm{pH}$ was 6.5 .

C314 is a water-soluble ester that is surface active. It was found from surface tension measurements that the surface excess of C314 did not exceed $5 \times 10^{13}$ molecules $/ \mathrm{cm}^{2}$ at the bulk saturation concentration of $30 \mu \mathrm{M}$. Experiments were typically run at a $28 \mu \mathrm{M}$ bulk concentration to maximize signal. No change in dynamics was observed with $14 \mu \mathrm{M}$ bulk concentration. This indicates that energy transfer and dimerizations were not factors in these experiments. $\mathrm{C} 314$ is ideal for $\mathrm{SH}$ spectroscopy using femtosecond Ti:sapphire lasers since the electronic origin provides a strong two-photon resonance enhancement to the $\mathrm{SH}$ from an $840 \mathrm{~nm}$ fundamental probe. C314 has an extinction coefficient of $45000 \mathrm{~L} / \mathrm{M} \mathrm{cm}$ which allows for substantial excitation by a low-power pump. The ground-state recovery time of C314 in bulk water was measured to be 4.7 ns by time-correlated single photon counting.

The TRSHG pump-probe setup is shown in Figure 2. A regeneratively amplified Ti:sapphire laser (Clark MXR) was used to generate $1 \mathrm{~mJ}$ energy, $100 \mathrm{fs}$ long duration, $840 \mathrm{~nm}$ wavelength pulses of light at $1 \mathrm{KHz}$. The pump excitation light at $420 \mathrm{~nm}$ was obtained by frequency doubling the fundamental. The $500 \mathrm{~nJ}$ pump was focused by a $1 \mathrm{~m}$ f.l. lens and directed to the sample at normal incidence to a $1 \mathrm{~mm}$ diameter spot for the orientational measurements. The pump intensity was at all times well below saturation. A half-wave plate followed by a quarter-wave plate (both zero order quartz at $420 \mathrm{~nm}$ ) were used to control the polarization. The linear polarizations were verified to be better than $300: 1$.

Part of the fundamental beam served as the probe beam. The probe was sent through a half-wave plate-polarizer attenuater and then focused onto the sample by a $1 \mathrm{~m} \mathrm{f}$.1. lens to a $1 \mathrm{~mm}$ spot diameter. The probe incidence angle was $70^{\circ}$ from normal. The polarization of the probe and the setting of the analyzer could be varied to select the desired tenser element of $\chi^{(2)}$. A colored long-wave pass glass filter was placed in the probe beam immediately before the sample to block any spurious source of $\mathrm{SH}$.

A polarizer was used to analyze the $\mathrm{SH}$ signal of the desired element of $\chi^{(2)}$. Finally, a colored short-wave pass glass filter was used to block any remaining fundamental, and the $\mathrm{SH}$ was focused into a 1/4 m monochromator (Jarrell Ash) and detected by a photomultiplier tube (R4220P, Hamamatsu). The signal from the PMT was averaged by a box car gated integrator (SRS) with a typical effective time constant of $300 \mathrm{~ms}$. Laser stability was excellent $(<1 \%$ shot to shot) so that shot-to-shot normalization was not necessary. The box car signal was digitized by a computer A/D board (National Instruments), and the $\mathrm{SH}$ was recorded as a function of pump delay by the computer (Apple Macintosh). Typically, 40 data sets were averaged at each pump polarization.

A shallow Teflon beaker was used to contain the C314 solution. This beaker was rotated at $1 \mathrm{rpm}$ with the probe spot $1 \mathrm{~cm}$ off axis in order to minimize any heating or other photoinduced process in the pump/probe region of the surface. No sample degradation was observed. The kinetics obtained upon reduction of power by $50 \%$ were identical.

\section{Results and Discussion}

4.1. Out-of-Plane Orientational Dynamics. The circularly pumped TRSHG kinetics for the out-of-plane orientational motion of coumarin $314,26 \mu \mathrm{M}$, at the water/air interface is shown in Figure 5. The pump was circularly polarized incoming normal to the interface at $420 \mathrm{~nm}, 100 \mathrm{~nJ}$. The probe was at

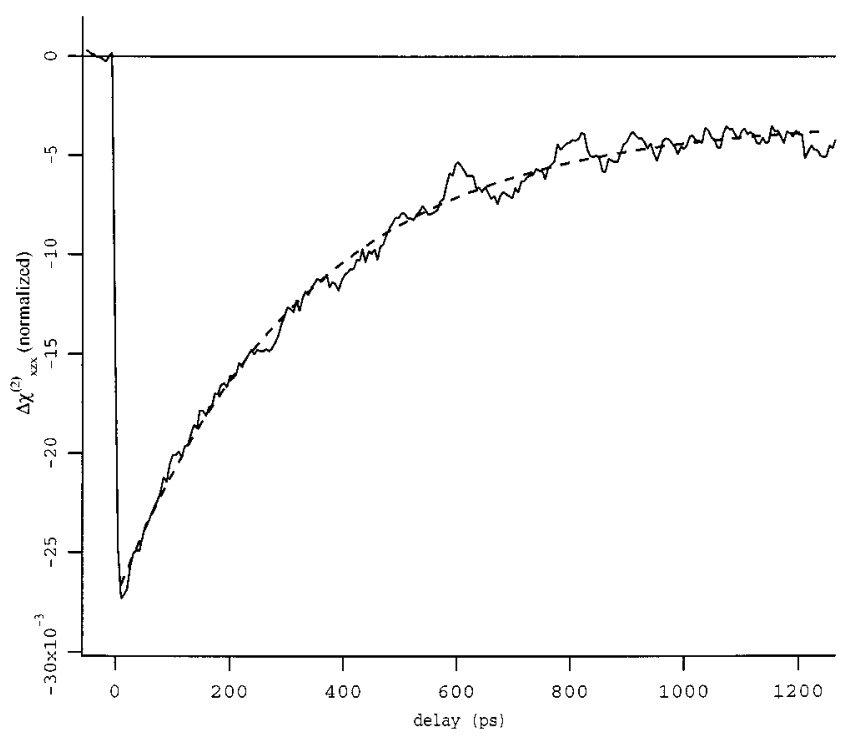

Figure 5. Circularly polarized pumping showing only the out-of-plane orientational motion of coumarin $314,26 \mu \mathrm{M}$, at the water/air interface. The $420 \mathrm{~nm}$ pump was circularly polarized incoming normal to the interface. The probe was at $840 \mathrm{~nm}$. The normalized second harmonic electric field at $420 \mathrm{~nm}$ for the element $\chi_{x z x}^{(2)}$ is plotted vs pump delay. The dashed line is a fit to a single exponential plus baseline giving an out of plane decay time of $350 \pm 20$ ps. The remaining $\sim 15 \%$ bleach after $1000 \mathrm{ps}$ is the electronic ground-state recovery.

$840 \mathrm{~nm}, 80 \mu \mathrm{J}$, at $70^{\circ}$ from normal, linearly polarized at $45^{\circ}$. The normalized second harmonic electric field at $420 \mathrm{~nm}$, analyzed at $90^{\circ}\left(\chi_{x z x}^{(2)}\right)$, is plotted vs pump delay. The amplitude of the electric field is the square root of the intensity. The dashed line is a fit to a single-exponential plus baseline giving an outof-plane decay time of $350 \pm 20$ ps. The signal-to-noise of the data was excellent, and the error in all fits is estimated to be less than $5 \%$.

The TRSHG kinetics recover from $t=0$ to $t=1 \mathrm{~ns}$ as the out-of-plane orientational motion redistributes the pumped C314 ensemble to the equilibrium orientational distribution. The remaining baseline of $15 \%$ bleach after $\mathrm{t} \approx 1000 \mathrm{ps}$ is the electronic ground-state recovery. Although the equilibrium orientational average has been regained, the population must still recover with the interface excited-state lifetime. While not shown, the C314 air/water interfacial ground-state recovery lifetime was fit from longer time scans to be $4.5 \mathrm{~ns}$, similar to the bulk value of $4.7 \mathrm{~ns}$, indicating that neither solvent friction nor interface polarity significantly affects the excited-state lifetime the coumarin.

4.2. In-Plane Orientational Dynamics. The results of $X$ and $\mathrm{Y}$ linearly polarized pumping are shown in Figure 6. Except for the pump polarization, the experimental conditions are identical to the circularly pumped case. Special care was taken to ensure that the pump energy was identical for both polarizations. The $\mathrm{X}$ pump decay was a fit to a single exponential plus baseline giving a decay time of $320 \pm 15$ ps. The Y pump decay was fit to a decay time of $460 \pm 15$ ps. The baseline $15 \%$ bleach after $1000 \mathrm{ps}$ is the electronic ground-state recovery, which, as predicted, is identical in amplitude to the circular polarized result.

The exponential fits shown in Figure 6 are included to illustrate that the differing dependence of $\chi_{x z x}^{(2)}(t)$ to $\mathrm{X}$ and $\mathrm{Y}$ linearly polarized pumping yields experimentally different kinetics. The $\mathrm{X}$ and $\mathrm{Y}$ decay times are not the in-plane decay time alone but include an out-of-plane contribution. Because of the differing contributions of the in- and out-of-plane 


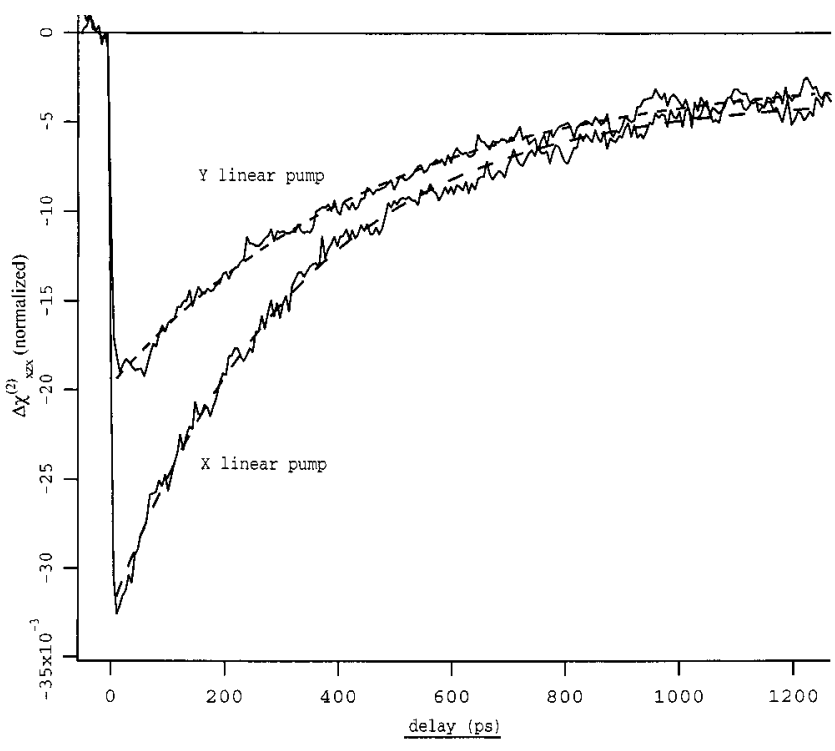

Figure 6. $\mathrm{X}$ (bottom) and $\mathrm{Y}$ (top) linearly polarized pumping showing the contribution of the in-plane orientational motion of coumarin 314 at the water/air interface to the TRSHG decay. The $420 \mathrm{~nm}$ pump was circularly polarized incoming normal to the interface. The probe was at $840 \mathrm{~nm}$. The normalized second harmonic electric field at $420 \mathrm{~nm}$ for the element $\chi_{x z x}^{(2)}$ is plotted vs pump delay. X pump: the dashed line is a fit to a single exponential plus baseline giving a decay time of 320 \pm 15 ps. Y pump: the dashed line is a fit with a decay time of $460 \pm$ 15 ps. The remaining bleach after 1000 ps is the electronic groundstate recovery, identical in amplitude to the circularly polarized result.

components, the data should not in principle be single exponential decays. However, because the in- and out-of-plane time constants are sufficiently close, they appear to be exponential within the experimental precision.

The X, Y, and circularly pumped data all recover to the same SH signal by 1000 ps. This indicates, as expected, that regardless of the initial photoselection due to pump polarization, the same equilibrium orientational distribution is reached. As a further check on the consistency of this treatment, we note that one can show that the average of the kinetic curves for the $\mathrm{X}$-polarized experiment and the Y-polarized experiment should be precisely equal to the circularly polarized kinetic curve. This is precisely what we obtain; namely, the average of the $\mathrm{X}$ and $\mathrm{Y}$ decay curve has a recovery time of $365 \pm 15 \mathrm{ps}$, which is in good agreement with the circularly polarized experiment result of $350 \pm 20$ ps.

The in-plane orientational dynamics were separated from the out-of-plane dynamics by the method described in section 2.6. The relative decay of the in-plane anisotropy given by eq 14 is shown in Figure 7A. This figure illustrates the in-plane orientational motion with the out-of plane components factored out. The dashed line through the data is a fit to an exponential decay with a time constant of $600 \pm 40$ ps.

We have performed additional pump-probe experiments on C314 at the air/water interface using sum-frequency generation (SFG) detection. The SFG experiments are beyond the scope of the current work, except that as a test, the orientational motion of C314 at the air/water interface was detected by SFG for X and $\mathrm{Y}$ linearly polarized pumping. In a sum-frequency experiment, the signal is generated from the mixing of two space and time coincident probe beams. The signal frequency $\omega_{3}$ is the sum of the two input frequencies $\omega_{2}$ and $\omega_{1}$ such that $\omega_{3}=\omega_{2}$ $+\omega_{1}$. For the orientational SFG experiment, the probe wavelengths were chosen to be 840 and $420 \mathrm{~nm}$, and the signal wavelength was $280 \mathrm{~nm}$. The pumping polarizations and
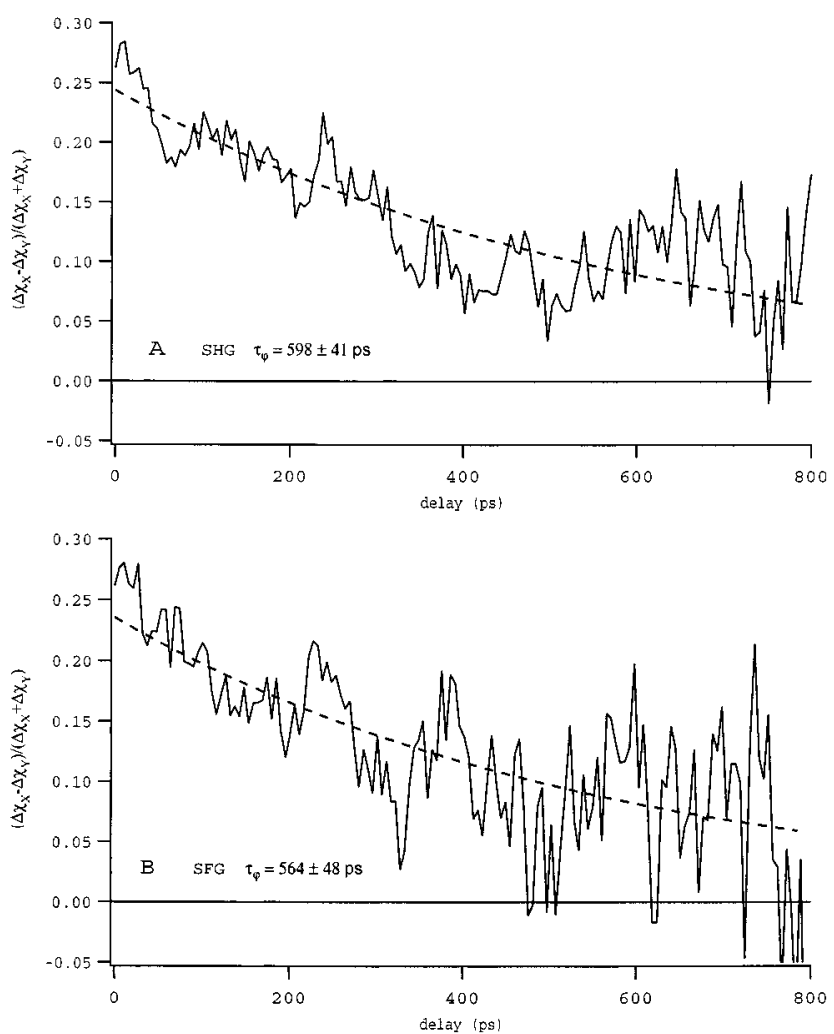

Figure 7. (A) SHG: relative decay of anisotropy illustrating in-plane $\varphi$ orientational motion alone, factoring out out-of-plane $\theta$ components, as derived from the second harmonic detected $\mathrm{X}$ and $\mathrm{Y}$ linear pump data (see Figure 6). The ratio $\left[\Delta \chi_{x z x: \mathrm{X}}^{(2)}(t)-\Delta \chi_{x z x: \mathrm{Y}}^{(2)}(t)\right] /\left[\Delta \chi_{x z x: \mathrm{X}}^{(2)}(t)+\right.$ $\left.\Delta \chi_{x z x: \mathrm{X}}^{(2)}(t)\right]$ in eq 14 is plotted vs pump delay. The dashed line through the data is a fit with a time constant of $600 \pm 40 \mathrm{ps}$. (B) SFG: relative decay of anisotropy illustrating in-plane $\varphi$ orientational motion alone, factoring out out-of-plane $\theta$ components, as derived from the sum frequency detected $\mathrm{X}$ and $\mathrm{Y}$ linear pump data (see text). The ratio $\left[\Delta \chi_{x x z: \mathrm{X}}^{(2)}(t)-\Delta \chi_{x x z: \mathrm{Y}}^{(2)}(t)\right] /\left[\Delta \chi_{x x z \mathrm{X}}^{(2)}(t)+\Delta \chi_{x x z: \mathrm{X}}^{(2)}(t)\right]$ in eq 14 is plotted vs pump delay. The dashed line through the data is a fit with a time constant of $560 \pm 50$ ps.

geometry were the same as the SH experiment.. The SFG susceptibility element $\chi_{x x z}^{(2)}\left(\omega_{3}, \omega_{2}, \omega_{1}\right)$ detected in this experiment had the same orientational dependence as the SH element $\chi_{x z x}^{(2)}\left(\omega_{2}, \omega_{1}, \omega_{1}\right)$. Therefore, the SFG detected kinetics of C314 at the air/water interface should be identical to the previous $\mathrm{SH}$ detected kinetics. This is indeed what was found. Figure 7B shows the in-plane $\varphi$ orientational motion given by eq 14 , factoring out out-of plane $\theta$ components, as derived from the SFG detected $\mathrm{X}$ and $\mathrm{Y}$ linear pump data. The dashed line through the data is a fit with a time constant of $560 \pm 50 \mathrm{ps,}$ giving excellent agreement with the value fit from the $\mathrm{SH}$ data of $600 \pm 40 \mathrm{ps}$. The out-of-plane decay time (not shown) was $340 \pm 20$ ps, also in good agreement with the SH result. The SFG experiment provides confirmation that the in-plane orientational decay time is in fact slower that the out-of-plane orientational decay time for $\mathrm{C} 314$ at the air/water interface.

4.3. Discussion. The bulk water isotropic orientational diffusion time for a coumarin with nearly identical hydrodynamic volume is 100 ps. ${ }^{36}$ However, for C 314 the out-of-plane relaxation time of $350 \mathrm{ps}$ and in-plane relaxation time of 600 ps are both over a factor of 3 slower than the rotations of a comparable coumarin in bulk water. The data show that the interfacial motions of $\mathrm{C} 314$ experience more friction than in the bulk. These results for $\mathrm{C} 314$ are similar to the previous results on Rh6G where the interfacial out-of-plane motions were likewise slower than the bulk orientational diffusion of Rh6G. ${ }^{16}$ 
We are currently developing a model relating the restricted surface orientational motions as measured by TRSHG to the bulk isotropic orientational diffusion. It is possible that the TRSHG measured kinetics of restricted motions are related by a different factor to the bulk orientational diffusion constant than the experimental methods that measure orientational diffusion times in the bulk. Note that this in no way affects the comparison of in- and out-of-plane motion from TRSHG data alone, only the comparison to bulk results.

Our measurements indicate that the in-plane reorientation time for C314 at the air/water interface is slower than the out-ofplane reorientation time. This result is consistent with the idea that the when the orientational distribution $\theta$ is perturbed there is a restoring force to return the $\theta$ distribution to the equilibrium asymmetric distribution. The in-plane motions have no in-plane, $\varphi$-dependent potential to respond to, only the in-plane friction. It is the additional restoring force for the $\theta$ motion that results in its being faster than that of the in-plane $\varphi$ motion.

In other studies it has been proposed that the extent to which structural parts of the solute protrude into the air versus the water region of the air/water interface affects the isomerization dynamics at the interface..$^{30,31}$ If the motions along the reaction coordinate extend significantly into the air phase, then the relaxation process may be faster at the interface than in the bulk even if the overall friction is greater in the water region of the surface than in the bulk region of the liquid. In the experiments reported here, $\mathrm{C} 314$ has slower interfacial orientation relaxation than in the bulk which indicates that its rotational motions both in and out of the interface plane are chiefly controlled by the friction on the water side of the interface.

Molecular dynamics treatments of the air/water interface predict that the interfacial friction should be less at the air/water interface than in the water bulk. ${ }^{37-39}$ However, these molecular dynamics simulations also show that the hydrogen bonds of the interfacial water last longer than those in the bulk. ${ }^{37-39}$ Both the current results on $\mathrm{C} 314$ and the previous results on $\mathrm{Rh} 6 \mathrm{G}^{16}$ which indicate slower interfacial orientational motion than in the bulk appear to conflict with decreased interfacial friction. However, the surface asymmetry orients the adsorbates and keeps their motions in a restricted geometry in a way that does not occur in the bulk. It is possible that both $\mathrm{C} 314$ and Rh6G possess an average orientation favorable to hydrogen bonding with the water network. The coupling to longer lived hydrogen bonds within the restricted range of motion could lead to slower orientational motions for these molecules at the air/water interface.

Unlike the coumarin and Rh6G molecules, for which an increase in the orientational diffusion time at the air/water interface relative to the bulk was observed, the orientational relaxation of eosin $\mathrm{B}$ was observed to undergo a decrease of the orientational relaxation time from 350 to 90 ps. ${ }^{17}$ The decreased relaxation time was attributed to reduced hydrogen bonding of water to eosin $\mathrm{B}$ due to increased competition with the interfacial water.

\section{Conclusions}

We have established through theory and method that timeresolved surface second harmonic generation can be used to detect both out-of-plane and in-plane orientational motions. The interfacial solute motions of coumarin 314 at the air/water interface were found to be anisotropic, with differing out-ofplane and in-plane reorientation time constants. We report the first direct observation of in-plane orientational motion of a molecule (coumarin 314) at the water/air interface using TRSHG. The in-plane reorientation time constant is $600 \pm 40$ ps. The out-of-plane reorientation time constant is $350 \pm 20$ ps. The surface reorientation times are 2-3 times slower than the bulk isotropic orientational diffusion time. The orientational motion of $\mathrm{C} 314$ is consistent with earlier results on rhodamine $6 \mathrm{G}$ at the air/water interface which indicated increased interfacial friction for these adsorbates compared with bulk aqueous solution.

Acknowledgment. The authors acknowledge the Department of Energy for their support and the National Science Foundation for equipment support. We also thank Prof. B. Berne for useful conversations.

\section{References and Notes}

(1) Heinz, T. F. Nonlinear Surface Electromagnetic Phenomena; Ponath, H., Stegeman, G., Eds.; Elsevier: Amsterdam, 1991.

(2) Eisenthal, K. B. Acc. Chem. Res. 1993, 26, 636.

(3) Eisenthal, K. B. Annu. Rev. Phys. Chem. 1992, 43, 627.

(4) Shen, Y. R. Аnпи. Rev. Phys. Chem. 1989, 40, 327.

(5) Richmond, G. L.; Robinson, J. M.; Shannon, V. L. Prog. Surface. Sci. 1988, 28, 1 .

(6) Bain, C. D.; Davies, P. B.; Ong, T. H.; Ward, R. N.; Brown, M. A. Langmuir 1991, 7, 1563.

(7) Berkovic, G. Physica A 1990, 168, 140.

(8) Corn, R. M.; Higgins, D. A. Chem. Rev. 1994, 94, 107.

(9) Hicks, J. M.; Kemnitz, K.; Eisenthal, K. B.; Heinz, T. F. J. Phys. Chem. 1986, 90, 560.

(10) Rasing, T.; Shen., Y. R.; Kim, M. W.; Valint, J. P.; Bock, J. Phys. Rev. A 1985, 31, 537

(11) Zhang, D.; Gutow, J. H.; Eisenthal, K. B.; Heinz, T. F. J. Chem Phys. 1993, 98, 5099 .

(12) Wang, H.; Borguet, E.; Eisenthal, K. B. J. Phys. Chem. A 1997, 101,713 .

(13) Wang, H.; Borguet, E.; Eisenthal, K. B. J. Phys. Chem. B 1998, 102,4927

(14) Zhao, X.; Subrahmanyan, S.; Eisenthal, K. B. Chem. Phys. Lett. $1990,171,558$

(15) Castro, A.; Bhattacharyya, K.; Eisenthal, K. B. J. Chem. Phys. 1991, 95,1310 .

(16) Castro, A.; Sitzman, E. V.; Zhang, D.; Eisenthal, K. B. J. Phys. Chem. 1991, 95, 6752.

(17) Antoine, R.; Tamburello-Luca, A. A.; Hebert, P. H.; Brevet, P. F.; Girault, H. H. Chem. Phys. Lett. 1998, 288, 138.

(18) Sitzmann, E. V.; Eisenthal, K. B. J. Chem. Phys. 1989, 90, 2831

(19) Eisenthal, K. B. J. Phys. Chem. 1996, 100, 12997.

(20) Shen, Y. R. The Principles of Nonlinear Optics; Wiley-Interscience: New York, 1984

(21) Brevet, P. F.; Girault, H. H. Second Harmonic Generation at Liquid/ Liquid Interfaces; CRC Press: Boca Rotan, FL, 1996.

(22) Mobius, D.; Ahuja, R. C.; Caminati, G.; Chi, L.; Cordroch, W.; Li, Z.; Masumoto, M. In Proc. Dyn. Mech. Pohotoinduced Electron Transfer Relat. Phenom.; Mataga, N., Okada, T., Msuhara, H., Eds.; NorthHolland: Amsterdam, 1992; p 377.

(23) Anfinrud, P. A.; Hart, D. E.; Hedstrom, J. F.; Struve, W. S. J. Phys Chem. 1986, 90, 5887.

(24) Worth, M. J.; Burbage, J. D. J. Phys. Chem. 1992, 96, 9022.

(25) Piasecki, D. A.; Wirth, M. J. J. Phys. Chem. 1993, 97, 7700

(26) Meech, S. R.; Yoshihara, K. Chem. Phys. Lett. 1990, 174, 423.

(27) Yanagimachi, M.; Tamai, N.; Masuhara, H. Chem. Phys. Lett. 1993 $201,115$.

(28) Morgenthaler, M. J. E.; Meech, S. R. J. Phys. Chem. 1996, 100, 3323.

(29) Meech, S. R.; Yoshihara, K. J. Phys. Chem. 1990, 94, 4913.

(30) Shi, X.; Borguet, E.; Tarnovsky, A. N.; Eisenthal, K. B. Chem. Phys. 1996, 205, 167.

(31) Sitzmann, E. V.; Eisenthal, K. B. J. Phys. Chem. 1988, 92, 2831.

(32) Sitzmann, E. V.; Eisenthal, K. B. J. Phys. Chem. 1988, 92, 4579.

(33) Heinz, T. F.; Tom, H. W. K.; Shen, Y. R. Phys. Rev. A 1983, 28 1883.

(34) Higgins, D. A.; Abrams, M. B.; Byerly, S. K.; Corn, R. M. Langmuir 1992, 8, 1994.

(35) Zhang, D. Studies of Dynamics and Structure at Liquid Interfaces by Second Harmonic and Sum Frequency Generation. Dissertation, Columbia University, New York, 1994.

(36) Horng, M. L.; Gardecki, J. A.; Maroncelli, M. J. Phys. Chem. A 1997, 101, 1030 .

(37) Michael, D.; Benjamin, I. J. Phys. Chem. B 1998, 102, 5145.

(38) Benjamin, I. Chem. Rev. 1996, 96, 1449.

(39) Benjamin, I. J. Chem. Phys. 1992, 97, 1432. 